\title{
Grey Scale Image Multi-Thresholding Using Moth-Flame Algorithm and Tsallis Entropy
}

\author{
Seifedine Kadry ${ }^{1}$, Venkatesan Rajinikanth ${ }^{2}$ \\ ${ }^{1}$ Department of Mathematics and Computer Science, Faculty of Science, Beirut Arab University, Lebanon \\ ${ }^{2}$ Department of Electronics and Instrumentation Engineering, St. Joseph's College of Engineering, Chennai, India
}

\begin{tabular}{l} 
ARTICLE INFO \\
\hline Article history's: \\
Received 25 December 2020, \\
Revised 26 December 2020, \\
Accepted 27 December 2020. \\
\end{tabular}

Keywords:

Thresholding,

Tsallis entropy,

Moth-Flame algorithm,

Performance evaluation,

Validation

\begin{abstract}
In the current era, image evaluations play a foremost role in a variety of domain, where the processing of the digital images is essential to identify the vital information. The image multi-thresholding is a vital image preprocessing field in which the available digital image is enhanced by grouping the similar pixel values. Normally, the digital test images are available in $\mathrm{RGB} /$ grey scale format and the appropriate processing methodology is essential to treat the images with a chosen methodology. In the proposed approach, Tsallis Entropy (TE) supported multi-level thresholding is planned for the benchmark grey scale imagery of dimension $512 \times 512 \times 1$ pixels using a chosen threshold values $(T=2,3,4,5)$. This work suggests the possible Cost Value $(\mathrm{CV})$ that can be considered during the optimization search and the proposed work is executed by considering the maximization of the TE as the $\mathrm{CV}$. The entire thresholding task is executed using Moth-Flame Algorithm (MFA) and the accomplished results are validated based on the image quality measures of various thresholds. The attained result with MFO is better compared to the result of CS, BFO, PSO and GA.
\end{abstract}

This work is licensed under a Creative Commons Attribution-Share Alike 4.0

\author{
Seifedine Kadry, \\ Faculty of Science, \\ Beirut Arab University, \\ Lebanon. \\ Email: skadry@gmail.com
}

\section{INTRODUCTION}

The recent literature confirms that the image processing methods are emerged as the key research domain because of its practical significance [1-5]. Normally, image processing methods will help to enhance the vital information in the image, which is further examined for information retrieval and decision making process. Even though a significant number of picture processing procedures are accessible in the literature; the thresholding methods are extensively implemented by the researchers to enhance and treat the information in digital photographs [6-10].

Normally, the thresholding methods are considered to enhancing the test images (RGB/Grey) by grouping the similar pixels; which will improve the information content in the trial image to be inspected. The bi-level thresholding is quite simple and it can be implemented manually, in which the operators are allowed to arbitrarily adjust the thresholds, till the required task is accomplished. When, a multi-level pixel grouping is needed; then the complexity of the process increases and this procedure needs a computer algorithm to assists the operator to select the finest value of the thresholds [11-13]. Hence, in modern days, a considerable quantity of automated threshold selection actions is proposed and implemented by the researchers to enhance the digital images of varied domains [14].

Image thresholding using the heuristic algorithm is a widely accepted procedure and various techniques to be followed during the threshold selection can be found in the recent research work [15-17]. The optimal selection of the threshold depends on; (i) The dimension of the image, (ii) Complexity in the pixel distribution, and the (iii) Nature of image (RGB/Grey). In the literature, a unique image threshold selection procedure does not exist and based on the need and the expertise, most of the researchers may adopt the between-class assisted 
thresholding and the entropy assisted thresholding [15]. The entropy based schemes are usually considered when the irregular/informative pixel group in the image is to be improved and this method considered the maximization of the entropy as the prime task during the threshold selection.

Earlier works in multi-thresholding confirms the implementation of entropy based methods, such as Kapur, Shannon, Tsallis, Rényi, etc [18]. Normally, the thresholding of an image is initiated with assigned Threshold (T) values of 2,3,4 and 5 and the considered heuristic algorithm is expected to find the required $T$ value by enhancing the test image by grouping the similar pixels. This research work considered the Tsallis Entropy (TE) based multi-thresholding procedure to improve the figure based on the elected threshold value $(\mathrm{T}=2$ to 5). The main merit of this approach is that, it will enhance the abnormal pixel group in the image by providing better value of the image quality measures as confirmed in earlier works [15] [19].

The selection of finest threshold by maximizing TE is experimentally investigated using well known benchmark images of dimension $512 \times 512 \times 1$ pixels and to minimize the computation complexity, the traditional Moth-Flame Algorithm (MFA) is implemented. The MFA is one of the recently developed nature inspired algorithm and proved its superiority in variety of engineering optimization problems; including image thresholding.

In the proposed work, traditional MFA with chosen algorithm parameters are considered to solve the considered optimization problem by maximizing the assigned Cost Value (CV). This work also suggests the selection and implementation of single and weighted sum of multiple CV to enhance the outcome from the processed image. After thresholding the image with $\mathrm{T}=2$ to 5 , a relative analysis is implemented to compute the essential Image-Quality-Measures (IQM) and based on the attained IQM, the performance of planned technique is established. All the experimental work is realized via MATLAB software and the attained result is offered and conversed.

The other sections of this research are organized as follows; Section 2 presents the Context, Section 3 depicts the methodology, Section 4 and 5 discusses the results and conclusion, respectively.

\section{RELATED WORK}

The multi-thresholding is a frequently approved picture pre-processing scheme to group the pixels in digital image based on the chosen threshold value. The choice of the image threshold selection depends chiefly on the need and type of the image to be processed. Table 1 summarizes the entropy supported threshold selection methods available in the literature. From Table 1, one can authenticate that the MFA based image processing methods are accepted to examine a variety of images. Further, the TE based image thresholding methods are also adopted to evaluate the traditional and medical images and the existing results of the previous study confirms that the TE is one of the choice to pre-process RGB/Grey scale images.

Table 1. Summary of entropy based multi-thresholding approaches

\begin{tabular}{|c|c|c|}
\hline Reference & Implemented methodology & Image category \\
\hline Agrawal, et al. [20] & $\begin{array}{l}\text { This work implements Cuckoo-Search algorithm and TS based } \\
\text { thresholding on } 512 \times 512 \times 1 \text { dimension images. }\end{array}$ & Grey image \\
\hline Zhang and $\mathrm{Wu}[21]$ & $\begin{array}{l}\text { Implementation of Artificial Bee Colony algorithm is discussed in } \\
\text { this work with a class of bench mark images. }\end{array}$ & Grey image \\
\hline Y. Wang, et al. [22] & $\begin{array}{l}\text { Implementation of Cooperative Pigeon-inspired optimization is } \\
\text { employed to perform the multi-thresholding task. }\end{array}$ & Grey image \\
\hline K.S. Manic, et al. [23] & $\begin{array}{l}\text { This work implemented the multi-thresholding with Firefly- } \\
\text { Algorithm }\end{array}$ & Grey image \\
\hline Naji Alwerfali, et al. [24] & $\begin{array}{l}\text { Implementation of Fuzzy-Tsallis entropy is discussed for a class of } \\
\text { chosen threshold value }\end{array}$ & Grey image \\
\hline $\begin{array}{l}\text { Khairuzzaman } \quad \text { and } \\
\text { Chaudhury [25] }\end{array}$ & $\begin{array}{l}\text { Implementation of MFA and Kapur's entropy based multi- } \\
\text { thresholding is discussed. }\end{array}$ & Grey image \\
\hline $\begin{array}{l}\text { M. Abd El Aziz, et. al. } \\
\text { [26] }\end{array}$ & $\begin{array}{l}\text { Hybrid Moth-Flame algorithm is implemented to enhance the } \\
\text { images. }\end{array}$ & Grey image \\
\hline H. Jia, et al., [27] & $\begin{array}{l}\text { Implementation of multi-thresholding for RGB image is executed } \\
\text { for various thresholds. }\end{array}$ & RGB image \\
\hline $\begin{array}{l}\text { A.E. Hassanien, et al., } \\
\text { [28] }\end{array}$ & Examination of tomato leaf disease is discussed with MFA & Disease images \\
\hline
\end{tabular}

In the proposed work, the grey image multi-thresholding is discussed using MFA and TE with the entropy maximization concept and the attained results are compared and validated against the earlier research work of [20]. This comparison authenticates that this scheme supports to reach improved entropy compared with other 
approaches attained with methods, such as Cuckoo-Search (CS), Particle-Swarm-Optimization (PSO), Bacterial-Foraging-Algorithm (BFA) and Genetic-Algorithm (GA).

\section{METHODS}

This part show the adopted research work and various operations performed with this method is clearly depicted in Figure 1. The proposed methodology helps to locate finest threshold for the considered trial image till the cost value is maximized. The MFA is initialized with the nominal parameters along with the cost value (TE) to be maximized. In this work, the MFA is allowed to alter the threshold of the test image till the TE is maximized. When the fined value of threshold is identified, then the scheme helps to provide the threshold image as the outcome. Finally, the test image is contrast to the threshold image and the important IQM is calculated to confirm the performance of this method. The result attained with the implemented method confirms that the MFA+TE based multi-thresholding helps to get a healthier result on the considered images with size $521 \times 521 \times 1$ pixels.

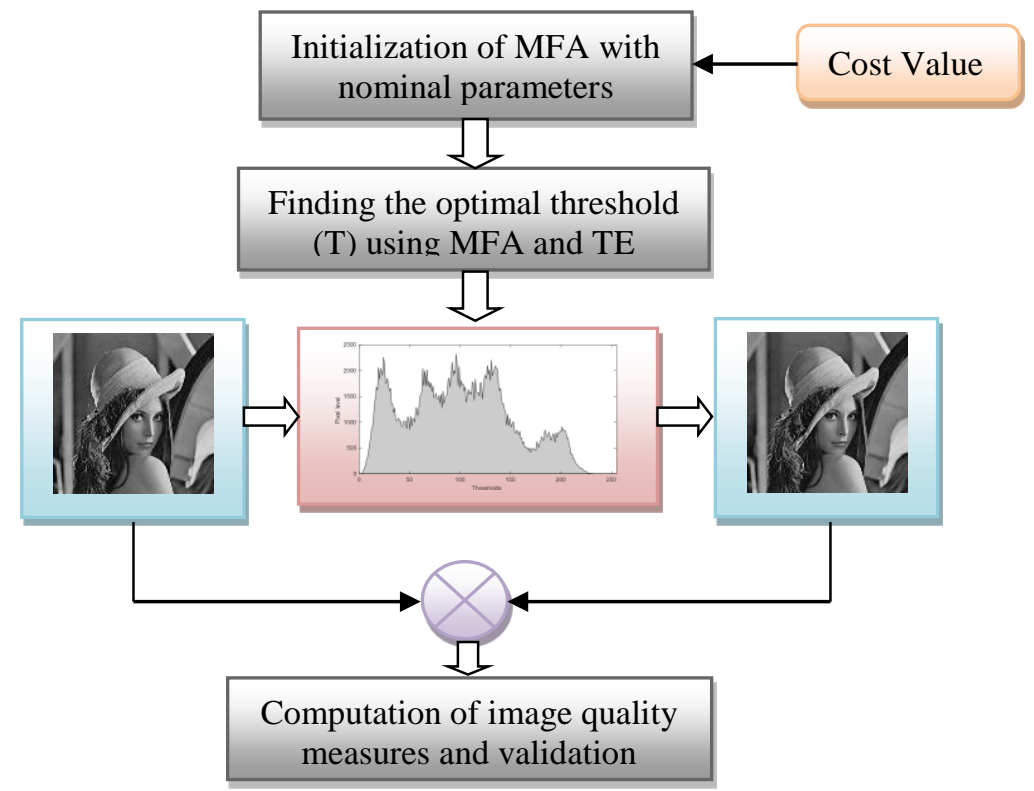

Fig. 1 Structure of proposed multi-thresholding scheme

\subsection{Tsallis Entropy}

The thresholding based on the entropy assisted approaches are commonly used in literature to pre-process digital imagery recorded with a class of modalities. TE is one of the methodologies adopted by the researches to enhance RGB/grey images [20-25].

The entropy is associated to the estimation of chaos in the image; Shannon initially calculated an entropy for finding the ambiguity regarding in images [29]. As per the statement by Shannon; when there is two statistically free sub-systems $\mathrm{E}_{1}$ and $\mathrm{E}_{2}$; then its entropy will be:

$$
\mathrm{S}_{\mathrm{h}}\left(\mathrm{E}_{1}+\mathrm{E}_{2}\right)=\mathrm{S}_{\mathrm{h}}\left(\mathrm{E}_{1}\right)+\mathrm{S}_{\mathrm{h}}\left(\mathrm{E}_{2}\right)
$$

Based on Eqn. (1), non-extensive entropy introduced by Tsallis can be defined as:

$$
S_{h_{I}}=\frac{1-\sum_{i=1}^{T}\left(B_{i}\right)^{I}}{I-1}
$$

where $T=$ threshold and $I=$ entropy index. Eqn. (2) will give Shannon's entropy for $I \rightarrow 1$.

The final entropy is:

$$
\mathrm{S}_{\mathrm{hI}}\left(\mathrm{E}_{1}+\mathrm{E}_{2}\right)=\mathrm{S}_{\mathrm{hI}}\left(\mathrm{E}_{1}\right)+\mathrm{S}_{\mathrm{h}} \mathrm{I}\left(\mathrm{E}_{2}\right)+(1-\mathrm{I}) \cdot \mathrm{S}_{\mathrm{hI}}\left(\mathrm{E}_{1}\right) \cdot \mathrm{S}_{\mathrm{hI}}\left(\mathrm{E}_{2}\right)
$$

Tsalli's is adopted to discover $T$ of the chosen image. Let the test image has $L$ gray values of span $\{0,1,2, \ldots, L\}$ with a probability functions; $\mathrm{B}_{\mathrm{i}}=\mathrm{B}_{0}, \mathrm{~B}_{1}, \ldots, \mathrm{B}_{\mathrm{L}-1} ;$ for $\mathrm{L}=256$. 
For the multi-level case, the TE is expressed as;

$$
\text { Tsallis }\left(t_{i}\right)=\left[t_{0}, t_{1}, \ldots, t_{L-1}\right]=\arg \max \left[S_{h I}^{E_{1}}(t)+S_{I}^{E_{2}}(t)+\ldots+S_{I}^{k}(t)+(1-I) \cdot S_{I}^{E_{1}}(t) \cdot S_{I}^{E_{2}}(t), \ldots, S_{I}^{E_{K}}(t)\right]
$$

where

$$
\begin{aligned}
& S_{h I}^{E_{1}}(t)=\frac{1-\sum_{i=0}^{t_{1}-1}\left(\frac{B i}{B^{E_{1}}}\right)^{I}}{I-1}, B^{E_{1}}=\sum_{i=0}^{t_{1}-1} B i \\
& \mathrm{~S}_{\mathrm{hI}}^{\mathrm{E}_{2}}(\mathrm{t})=\frac{1-\sum_{\mathrm{i}=\mathrm{t}_{1}}^{\mathrm{t}_{2}-1}\left(\frac{\mathrm{Bi}}{\mathrm{B}^{\mathrm{F}_{2}}}\right)^{\mathrm{I}}}{\mathrm{I}-1}, \mathrm{~B}^{\mathrm{E}_{2}}=\sum_{\mathrm{i}=\mathrm{t}_{1}}^{\mathrm{t}_{2}-1} \mathrm{Bi} \\
& S_{I}^{E_{k}}(t)=\frac{1-\sum_{i=t_{k}}^{L-1}\left(\frac{B i}{B^{k}}\right)^{I}}{I-1}, B^{E_{K}}=\sum_{i=t_{k}}^{L_{2}-1} B i
\end{aligned}
$$

Subject to the following constraints:

$$
\begin{array}{r}
\left|\mathrm{B}^{\mathrm{E}_{1}}+\mathrm{B}^{\mathrm{E}_{2}}\right|-1<\mathrm{S}_{\mathrm{h}}<1-\mathrm{B}\left|\mathrm{P}^{\mathrm{E}_{1}}-\mathrm{B}^{\mathrm{E}_{2}}\right| \\
\quad\left|\mathrm{B}^{\mathrm{E}_{2}}+\mathrm{B}^{\mathrm{E}_{3}}\right|-1<\mathrm{S}_{\mathrm{h}}<1-\left|\mathrm{B}^{\mathrm{E}_{2}}-\mathrm{B}^{\mathrm{E}_{3}}\right| \\
\mathrm{B}\left|\mathrm{P}^{\mathrm{E}_{\mathrm{k}}}+\mathrm{B}^{\mathrm{E}_{\mathrm{L}-1}}\right|-1<\mathrm{S}_{\mathrm{h}}<1-\left|\mathrm{B}^{\mathrm{E}_{\mathrm{k}}}-\mathrm{B}^{\mathrm{E}_{\mathrm{L}-1}}\right|
\end{array}
$$

The proposed methodology identifies the TE by varying the threshold vales.

\subsection{Cost Value}

The Cost Value (CV) is one of the major components in the proposed thresholding operation and the overall quality of the result depends on the $\mathrm{CV}$ considered in this approach. In this work, a single $\mathrm{CV}_{1}$ is considered to threshold the images. Further, this work also recommends the need for the weighted sum of CV $\left(\mathrm{CV}_{1} \& \mathrm{CV}_{2}\right)$ as depicted in Eqn. (8) and (9),

$$
\begin{aligned}
& \mathrm{CV}_{1}=\text { Tsallis }\left(\mathrm{t}_{\mathrm{i}}\right)_{\max }=\left[\mathrm{t}_{0}, \mathrm{t}_{1}, \ldots, \mathrm{t}_{\mathrm{L}-1}\right]_{\max } \\
& \mathrm{CV}_{2}=\mathrm{W} 1 * \text { Tsallis }\left(\mathrm{t}_{\mathrm{i}}\right)_{\text {max }}+\mathrm{W} 2 * \mathrm{PSNR}+\mathrm{W} 3 * \mathrm{SSIM} \\
& \mathrm{CV}_{3}=\mathrm{W} 1 * \text { Tsallis }\left(\mathrm{t}_{\mathrm{i}}\right)_{\text {max }}+\mathrm{W} 2 * \mathrm{PSNR}+\mathrm{W} 3 * \mathrm{SSIM}+\mathrm{W} 4 * \mathrm{FSM}
\end{aligned}
$$

In this work, the weights $\mathrm{W} 1$ to $\mathrm{W} 4$ are assigned as; $0<\mathrm{W}<1$ based on the priority and the earlier work clearly depicts the choice of the weight for the chosen problem [30].

\subsection{Moth-Flame Algorithm}

MFA proposed in 2015 [31] and the earlier works with this technique can be found in [25][26][28]. The background of MFO is according to the progress of a Moth to a Flame in an environment. In MFA, the exploration agents are the moths and the solution for the problem is the flame. Every moth is permitted to find the flame when the search iteration increases.

Let, a selected exploration location has A-numbers of moths $(\mathrm{M})$ and B-number of flames $(\mathrm{F})$. Let the beginning coldness between $\mathrm{M}$ and $\mathrm{F}$ is $\mathrm{D}_{\mathrm{A}}=\left|\mathrm{F}_{\mathrm{B}}-\mathrm{M}_{\mathrm{A}}\right|$, which is to be minimized to locate the result for the problem.

The movement of $\mathrm{M}$ towards $\mathrm{F}$ can be expressed as in Eqn. (10);

$$
\mathrm{M}_{\mathrm{A}}=\mathrm{D}_{\mathrm{A}} * \mathrm{e}^{\kappa \Re} * \cos (2 \pi \Re)+\mathrm{F}_{\mathrm{B}}
$$

where $\mathrm{M}_{\mathrm{A}}=\mathrm{A}^{\text {th }}$ moth, $\mathrm{F}_{\mathrm{B}}=\mathrm{B}^{\text {th }}$ flame, $\kappa=$ spiral pattern constant, and $\mathfrak{R}=$ random number $[-1,1]$. Other information on the MFA can be found in [25,26,31]. 
Figure 2 depicts the traced made by a $\mathrm{M}$ towards the $\mathrm{F}$. The following parameters are considered for the MAF; amount of $\mathrm{M}=30$, investigate dimension $=\mathrm{T}$ ( 2 to 5 ), maximum iteration (Iter $\left.r_{\max }\right)=5000$ and stopping criteria $=$ Iter $_{\max }$ or $\mathrm{CV}_{1}$.

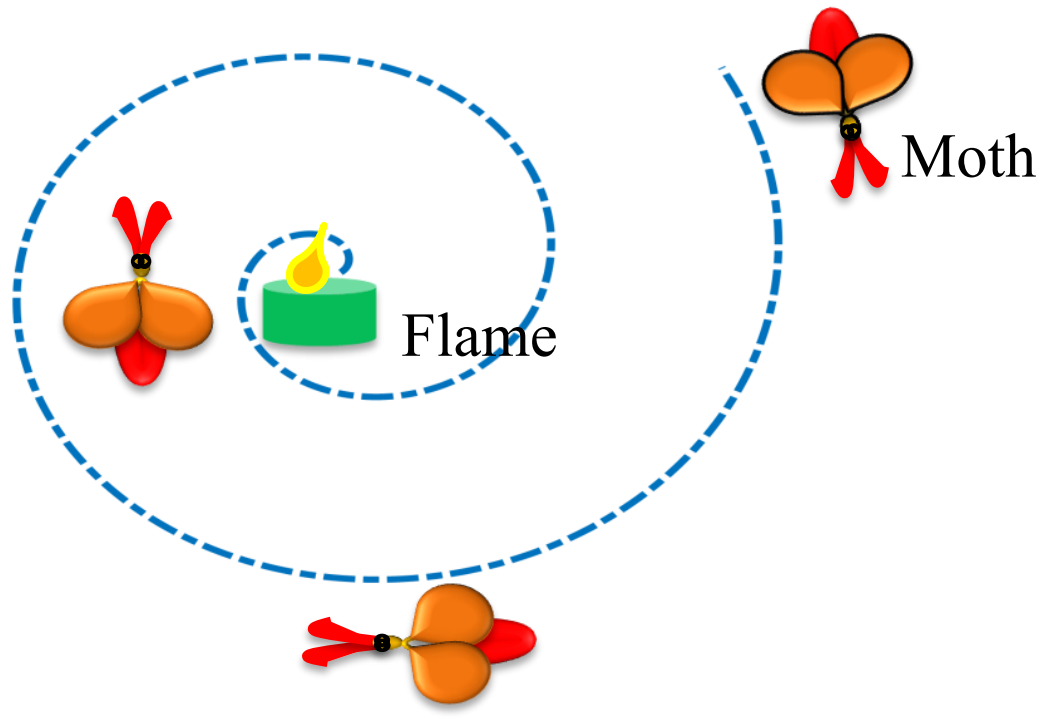

Fig. 2 Traces made by an artificial moth to a flame

\subsection{Performance Evaluation and Validation}

The merit of proposed scheme is computed based on the IQM obtainable in literature. In this work, the comparison among the test image and threshold image is executed to find the essential IQM, such as RootMean-Squared-Error (RMSE), Peak Signal-to-Noise Ratio (PSNR), Feature Similarity Index (FSI), StructuralSimilarity-Index-Measure (SSIM), Normalized-Cross-Correlation (NCC), Average-Difference (AD) and Structural-Content (SC) were computed and the information regarding this measures can be found in [20-24, 32].

\section{RESULTS AND DISCUSSION}

This section demonstrates the investigational outcome of MFA+TE based multi-thresholding attained for a class of benchmark images. The proposed experimental investigation is implemented using MATLAB software using a work station having configuration of Corei5, 16GB RAM and 2GB VRAM. The performance of this research is evaluated based on the CV hence the computation of CPU run time is not performed.

This work considered the well-known benchmark grey test images illustrated in Figure 3 for the assessment. Figure 3(a) presents the image and Figure 3(b) shows the grey-histogram. From the histogram, it can be known that, $\operatorname{Im} 1$ and $\operatorname{Im} 2$ is having oscillatory pixel distribution, $\operatorname{Im} 3$ and $\operatorname{Im} 4$ presents smooth pixel distribution and Im5 and Im6 depicts more oscillatory pixel distribution. Identification of finest threshold in the varied pixel distribution is quite complex compared to smooth distribution and this problem can be solved using the heuristic algorithm assisted threshold selection operation. In this work, MFA+TE is considered to find the thresholds for $\mathrm{T}=2$ to 5 and the results are presented and discussed to justify the need for the proposed research work.

Figure 4 depicts the identified threshold values on the histogram of $\operatorname{Im} 1$ for various thresholds. In this work, based on the assigned threshold, the MFA+TE groups the similar pixels of the image and the attained thresholds for the chosen image is clearly presented using Figure 4(a) to Figure 4(d).

After collecting all the threshold images for the chosen test image, a comparative assessment is then implemented to compute the important IQM to confirm the merit of proposed technique. This comparison helped to get various quality parameters including the SSIM. Figure 5 depicts the SSIM map attained for Im2 for $\mathrm{T}=2$ to 5 and these images are represented with "Parula Color map" for better visibility. From Fig.5(a) to (d), it can be noted that the MFA+TE supported thresholding helped to accomplish an improved SSIM map irrespective of the selected threshold value.

The thresholding results achieved for other images are depicted in Figure 6 for different thresholds as depicted in Figure 6(a) to Figure 6(d) and these outcomes authenticate that this method helps to achieve 
enhanced pixel grouping for the chosen threshold. The comparison between the test and threshold images is then executed and the computed IQM are presented for further assessment.
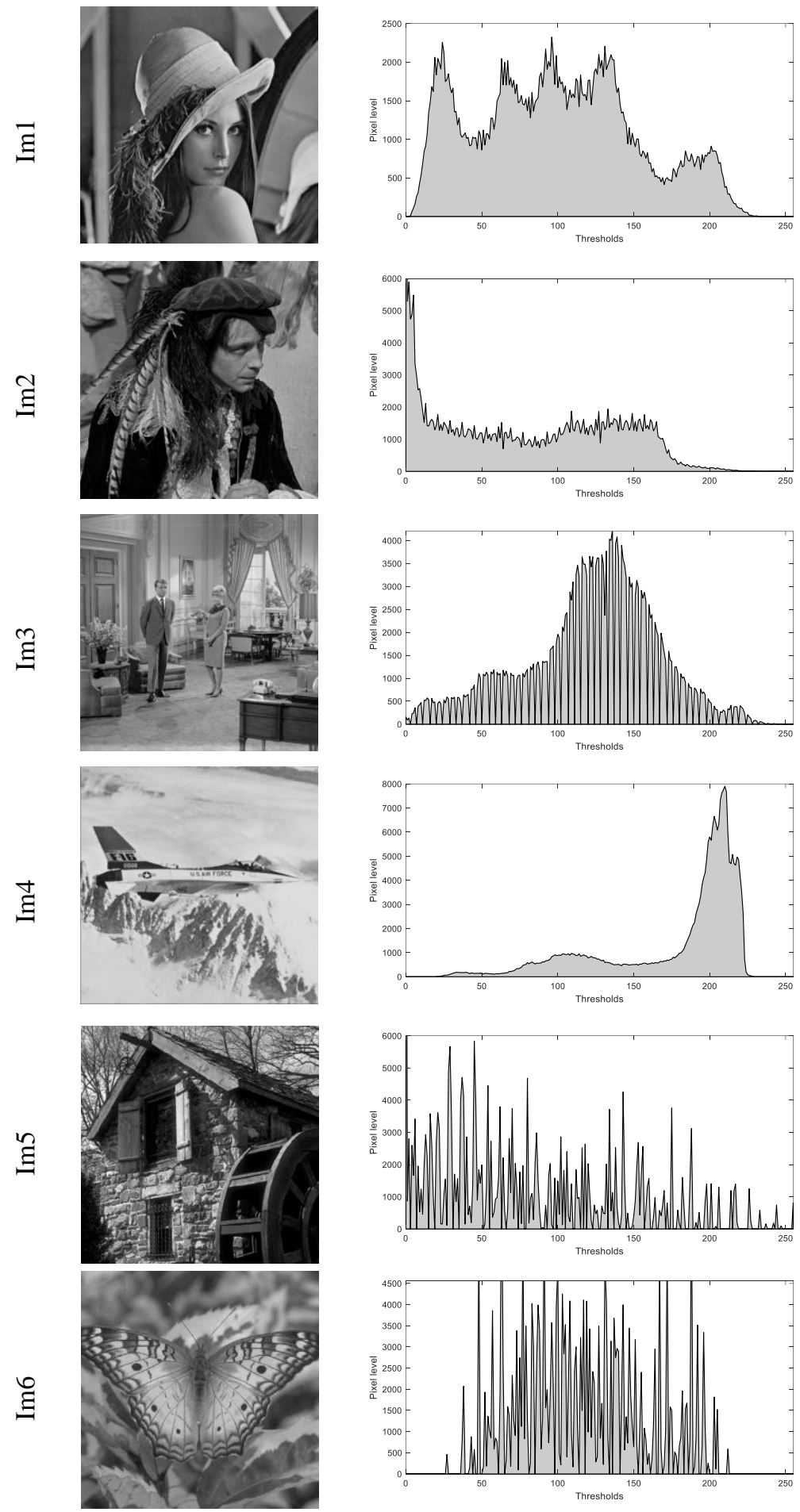

(a) Image

(b) Histogram

Fig. 3 Considered test images and the related histogram

Table 2 presents the computed IQM for all the considered images and the results in table prove that the image quality improves when the threshold-level increases. This condition also verified using the IQM of Im1 as depicted in Figure 7. The Glyph-plot presented in Figure 7 confirms that the IQM attained with T=5 is better compared to other values. 


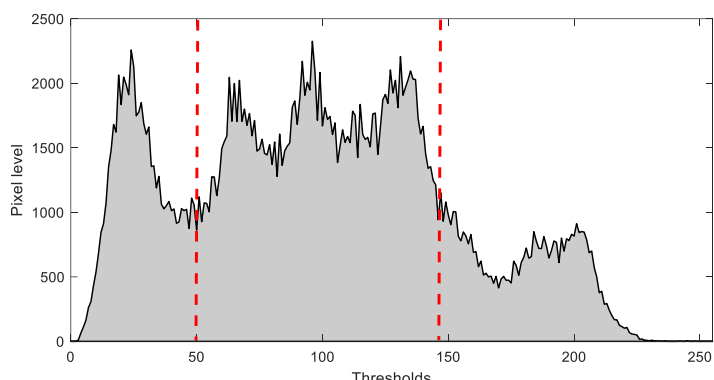

(a) $\mathrm{T}=2$

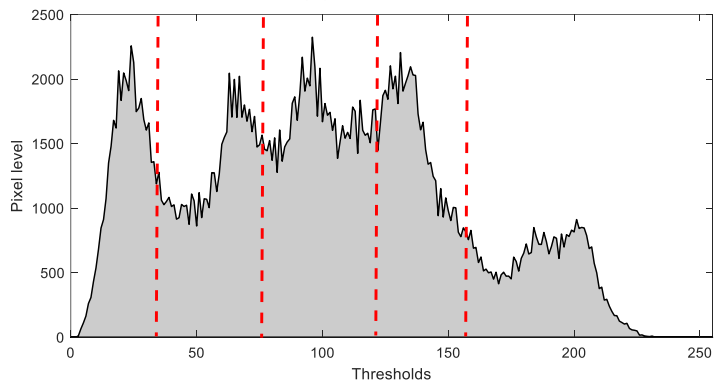

(c) $\mathrm{T}=4$

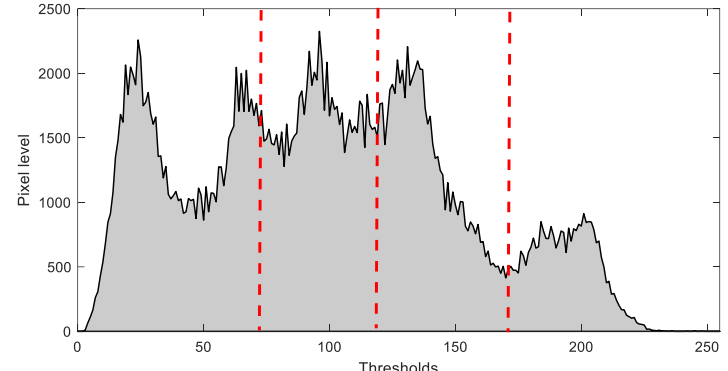

(b) $\quad \mathrm{T}=3$

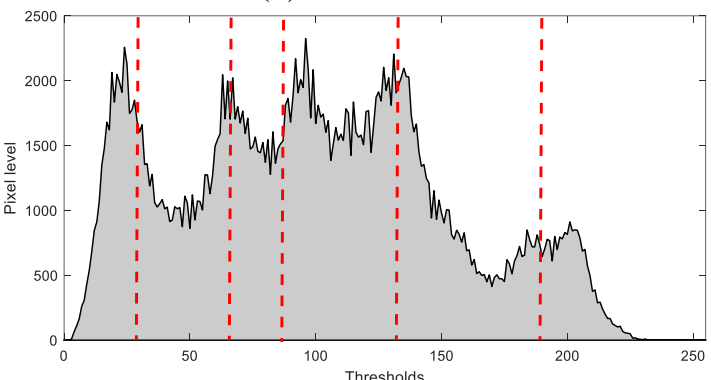

(d) $\mathrm{T}=5$

Fig. 4 Optimal thresholds attained with MFA+TE for $\mathrm{T}=2$ to 5

Table 2. Computed performance values for the thresholding results attained with MFA+TE

\begin{tabular}{ccccccccccc}
\hline Image & T & Entropy & NAE & RMSE & PSNR & FSM & SSIM & NCC & AD & SC \\
\hline & 2 & 1.1686 & 0.3276 & 37.6991 & 16.6042 & 0.6561 & 0.5841 & 1.0876 & -7.7274 & 0.7765 \\
$\Xi$ & 3 & 1.8461 & 0.2724 & 31.8142 & 18.0784 & 0.7672 & 0.6527 & 1.1372 & -6.3531 & 0.7381 \\
& 4 & 2.0681 & 0.1933 & 23.4481 & 20.7286 & 0.8027 & 0.7060 & 1.0548 & -0.2091 & 0.8671 \\
& 5 & 2.3848 & 0.1735 & 20.5615 & 21.8697 & 0.8499 & 0.7518 & 1.0543 & 0.9089 & 0.8755 \\
& 2 & 1.0147 & 0.3342 & 33.6252 & 17.5975 & 0.6759 & 0.4833 & 0.8536 & 17.2293 & 1.2093 \\
$\Xi$ & 3 & 1.5804 & 0.2454 & 23.2044 & 20.8194 & 0.7830 & 0.6486 & 1.0026 & 3.7815 & 0.9415 \\
& 4 & 1.9497 & 0.2703 & 26.8235 & 19.5605 & 0.8416 & 0.6977 & 1.1778 & -8.4175 & 0.6985 \\
& 5 & 2.0561 & 0.1644 & 16.3231 & 23.8747 & 0.8859 & 0.7232 & 0.9686 & 7.1931 & 1.0359 \\
& 2 & 0.8115 & 0.2196 & 32.3099 & 17.9441 & 0.6192 & 0.5524 & 0.9506 & 5.8383 & 1.0382 \\
$\Xi$ & 3 & 1.3982 & 0.1957 & 27.1626 & 19.4514 & 0.7391 & 0.6977 & 1.0745 & -8.3565 & 0.8383 \\
$\Xi$ & 4 & 1.8347 & 0.1713 & 25.7641 & 19.9105 & 0.8131 & 0.7725 & 1.1256 & -13.6304 & 0.7748 \\
& 5 & 2.0909 & 0.1181 & 17.3201 & 23.3598 & 0.8656 & 0.8170 & 1.0212 & 0.3028 & 0.9433 \\
& 2 & 0.9688 & 0.2440 & 46.6671 & 14.7506 & 0.7641 & 0.7999 & 1.2133 & -37.0899 & 0.6710 \\
$\Xi$ & 3 & 1.2258 & 0.2323 & 45.2031 & 15.0274 & 0.8183 & 0.8332 & 1.2143 & -35.6559 & 0.6718 \\
$\Xi$ & 4 & 1.7005 & 0.1949 & 39.3235 & 16.2378 & 0.8013 & 0.7590 & 1.1392 & -19.6490 & 0.7554 \\
& 5 & 1.9192 & 0.1512 & 32.1751 & 17.9804 & 0.8449 & 0.7982 & 1.1460 & -24.3337 & 0.7562 \\
& 2 & 1.3255 & 0.4044 & 37.8893 & 16.5605 & 0.7606 & 0.6062 & 1.0956 & -5.0505 & 0.7477 \\
$\Xi$ & 3 & 1.8051 & 0.3404 & 32.9350 & 17.7776 & 0.8362 & 0.6800 & 1.1828 & -7.3188 & 0.6775 \\
$\Xi$ & 4 & 2.1170 & 0.3284 & 32.4212 & 17.9142 & 0.8713 & 0.7848 & 1.2368 & -16.4717 & 0.6327 \\
& 5 & 2.4040 & 0.3019 & 29.6634 & 18.6864 & 0.9006 & 0.8056 & 1.2307 & -14.0050 & 0.6448 \\
& 2 & 0.7581 & 0.2975 & 40.2261 & 16.0406 & 0.5486 & 0.5016 & 1.0869 & -14.7631 & 0.7821 \\
ఏ & 3 & 1.6302 & 0.2205 & 31.8833 & 18.0595 & 0.7089 & 0.6803 & 1.1204 & -9.9773 & 0.7653 \\
& 4 & 1.7701 & 0.1949 & 28.4297 & 19.0554 & 0.7478 & 0.7168 & 1.0506 & -1.0068 & 0.8668 \\
& 5 & 2.2202 & 0.2150 & 29.8904 & 18.6202 & 0.7936 & 0.7502 & 1.1110 & -6.9498 & 0.7813 \\
\hline
\end{tabular}

The significance of the MFA+TE based thresholding is also verified using the CV (TE) of implemented scheme with the similar existing method in the literature as shown in Table 3. This prove that the CV attained using the MFA is better compared to CS, BFO, PSO and GA. This confirms that the MFA+TE halps to achieve better result for a classof grey images. 


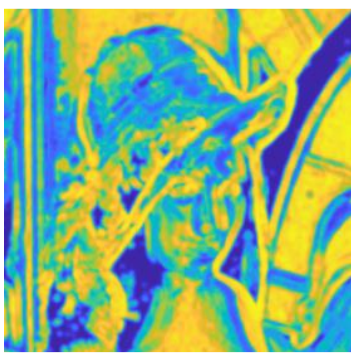

(a) $\mathrm{T}=2$

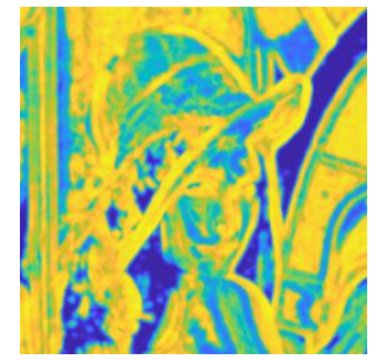

(b) $\mathrm{T}=3$

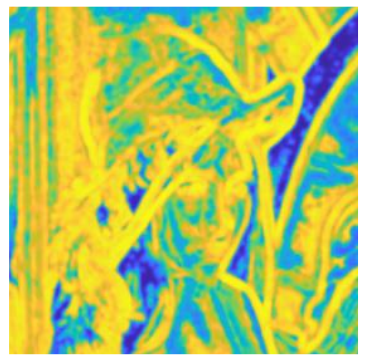

(c) $\mathrm{T}=4$

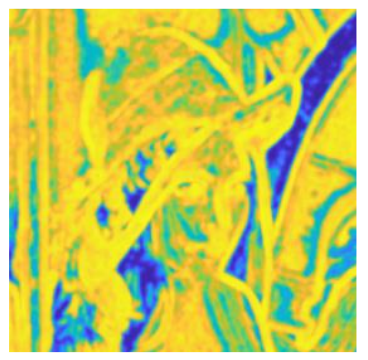

(d) $\mathrm{T}=5$

Fig. 5 Structural similarity map for $\mathrm{T}=2$ to 5

Table 3. Comparison of the TE attained with various heuristic algorithms

\begin{tabular}{|c|c|c|c|c|c|c|}
\hline Image & $\mathbf{T}$ & $\mathrm{CS}[20]$ & BFO [20] & PSO [20] & GA [20] & MFA \\
\hline \multirow{4}{*}{$\Xi$} & 2 & 0.8890 & 0.8889 & 0.8889 & 0.8889 & 1.1686 \\
\hline & 3 & 1.2963 & 1.2963 & 1.2963 & 1.2963 & 1.8461 \\
\hline & 4 & 1.6543 & 1.6543 & 1.6543 & 1.6543 & 2.0681 \\
\hline & 5 & 1.9959 & 1.9958 & 1.9958 & 1.99587 & 2.3848 \\
\hline \multirow{4}{*}{ ఏี } & 2 & 0.8889 & 0.8889 & 0.8889 & 0.8889 & 1.0147 \\
\hline & 3 & 1.2963 & 1.2963 & 1.2963 & 1.2962 & 1.5804 \\
\hline & 4 & 1.6543 & 1.6543 & 1.6543 & 1.6542 & 1.9497 \\
\hline & 5 & 1.9959 & 1.9958 & 1.9957 & 1.9957 & 2.0561 \\
\hline \multirow{4}{*}{$\stackrel{\varrho}{\Xi}$} & 2 & 0.8888 & 0.8888 & 0.8888 & 0.8888 & 0.8115 \\
\hline & 3 & 1.2963 & 1.2963 & 1.2963 & 1.2963 & 1.3982 \\
\hline & 4 & 1.6543 & 1.6543 & 1.6542 & 1.6542 & 1.8347 \\
\hline & 5 & 1.9958 & 1.9957 & 1.9957 & 1.9956 & 2.0909 \\
\hline \multirow{4}{*}{$\stackrel{\Xi}{\Xi}$} & 2 & 0.8889 & 0.8889 & 0.8889 & 0.8889 & 0.9688 \\
\hline & 3 & 1.2963 & 1.2962 & 1.2962 & 1.2962 & 1.2258 \\
\hline & 4 & 1.6543 & 1.6543 & 1.6543 & 1.6542 & 1.7005 \\
\hline & 5 & 1.9959 & 1.9958 & 1.9958 & 1.9958 & 1.9192 \\
\hline \multirow{4}{*}{$\stackrel{\curvearrowleft}{\Xi}$} & 2 & 0.8888 & 0.8888 & 0.8888 & 0.8888 & 1.3255 \\
\hline & 3 & 1.2963 & 1.2961 & 1.2961 & 1.2961 & 1.8051 \\
\hline & 4 & 1.6543 & 1.6536 & 1.6536 & 1.6536 & 2.1170 \\
\hline & 5 & 1.9959 & 1.9942 & 1.9937 & 1.9934 & 2.4040 \\
\hline \multirow{4}{*}{$\stackrel{\varrho}{\Xi}$} & 2 & 0.8889 & 0.8888 & 0.8888 & 0.8888 & 0.7581 \\
\hline & 3 & 1.2963 & 1.2962 & 1.2962 & 1.2962 & 1.6302 \\
\hline & 4 & 1.6543 & 1.6534 & 1.6526 & 1.6526 & 1.7701 \\
\hline & 5 & 1.9959 & 1.9948 & 1.9915 & 1.9894 & 2.2202 \\
\hline
\end{tabular}

The result of the proposed study confirms the merit of the MFA+TE based thresholding. In future, the multi-thresholding operation can be implemented using the CV proposed in Eqn. (8) and (9) and the attained IQM are compared and validated with other existing methods in the literature. Further, the performance of TE can be authorized with other entropy techniques, such as Kapur, Shannon and Rényi.

\section{CONCLUSION}

The proposed research work implemented MFA+TE based multi-thresholding for a class of benchmark imagery of dimension $512 \times 512 \times 1$ pixels. In this work; the maximization of TE is chosen as the CV and the image thresholding is implemented for $\mathrm{T}=2$ to 5 . In this work the traditional MFA is implemented to find the finest threshold for the considered test images by maximizing the TE. After obtaining the thresholded image, a comparison among the test image and the processed image is implemented to compute the essential IQM and based on the attained values of these IQMs, the performance of proposed approach is validated. The comparison of MFA with CS, PSO, BFO and GA confirms that the TE attained with MFA+TE is better contrast to the proposed work and this confirms the superiority of the MFA+TE thresholding. 

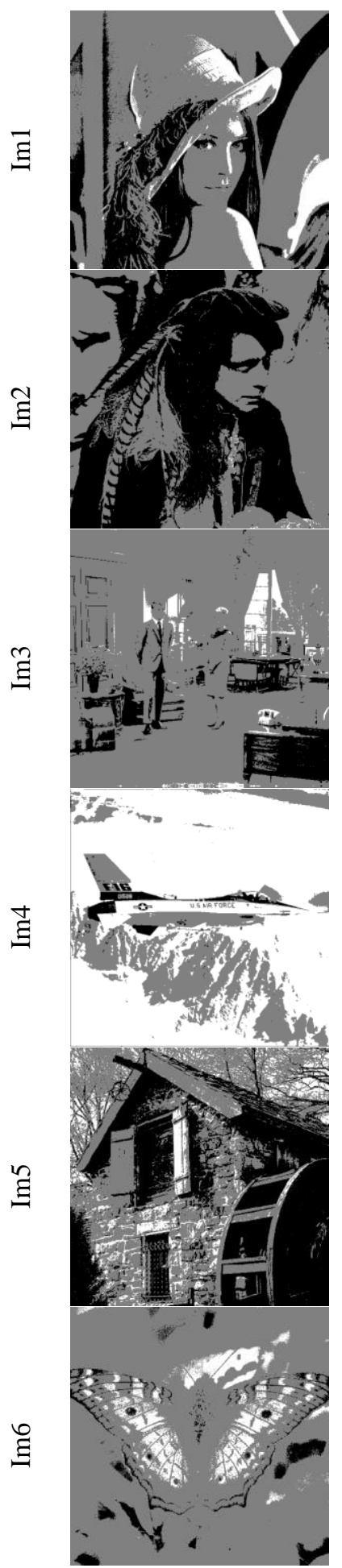

(a) $\mathrm{T}=2$
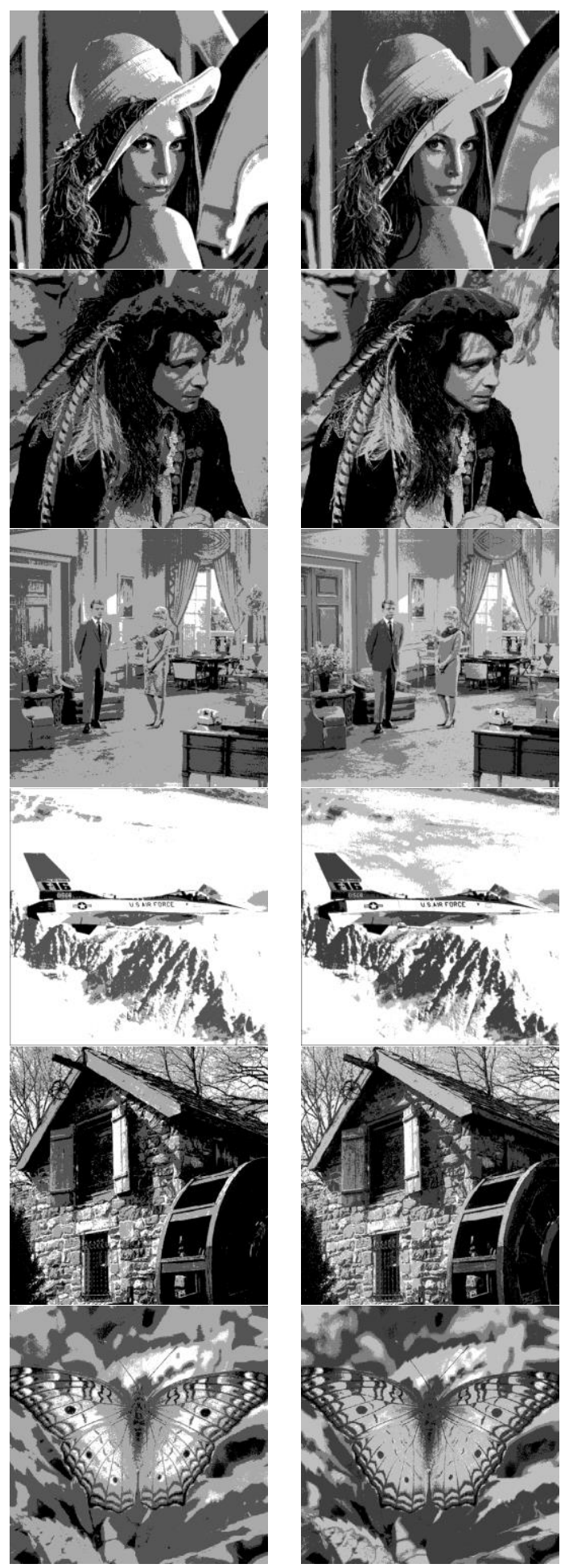

(b) $\mathrm{T}=3$

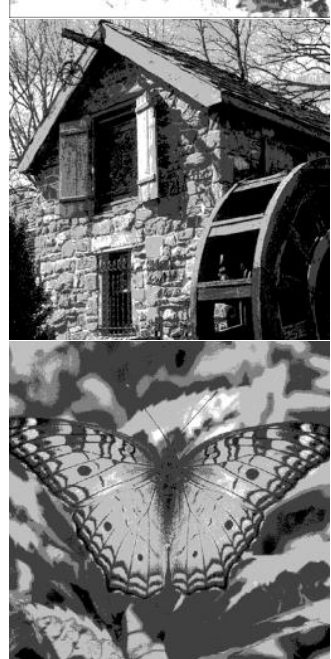

(c) $\mathrm{T}=4$
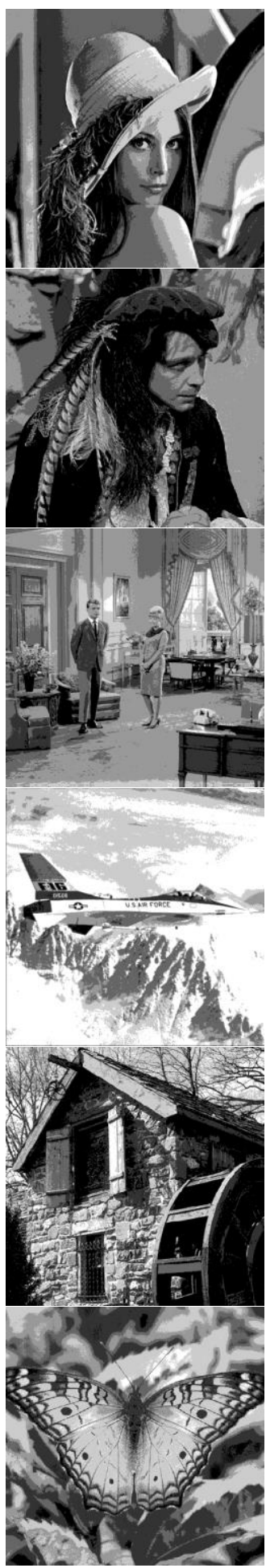

(d) $\mathrm{T}=5$

Fig. 6 The thresholding putcome attained with MFA+TE 


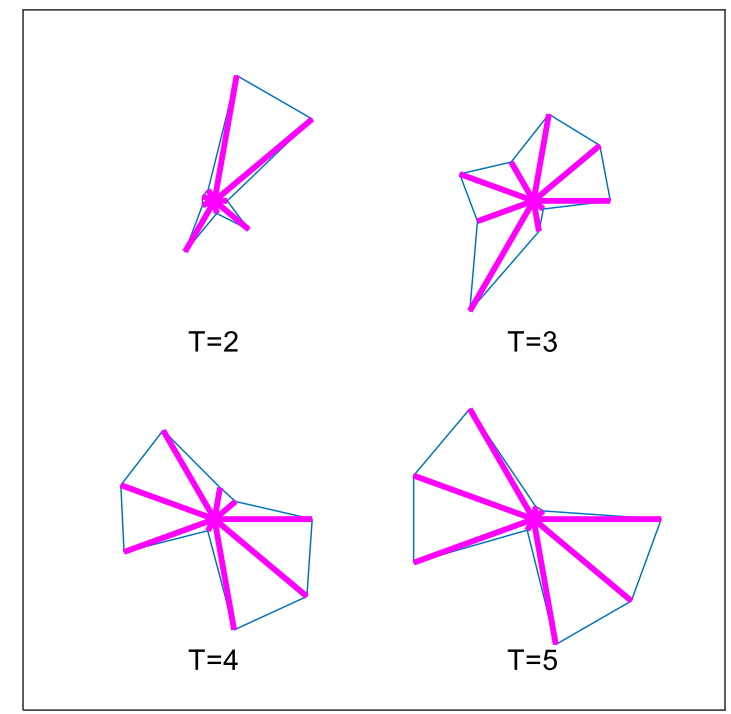

Fig. 7 Glyph-plot to demonstrate the improvement in performances with increase in threshod level

\section{REFERENCES}

[1] B. Muthu, C.B. Sivaparthipan, G. Manogaran, R. Sundarasekar, S. Kadry, A. Shanthini, and A. Dasel, "IOT Based Wearable Sensor for Diseases Prediction and Symptom Analysis in Healthcare Sector," Peer-to-peer networking and applications, pp.1-12, 2020. DOI: https://doi.org/10.1007/s12083-019-00823-2

[2] F. Kulakov, S. Kadry, G. Alferov, and A. Sharlay, "Bilateral Remote Control Over Space Manipulators," In AIP Conference Proceedings, vol. 2040, no. 1, pp. 150015, 2018. DOI: https://doi.org/10.1063/1.5079218

[3] A. Madi, O.K. Zein, and S. Kadry., "On the Improvement of Cyclomatic Complexity Metric,” International Journal of Software Engineering and Its Applications, vol.7, no.2, pp.67-82, 2013.

[4] A. Bakiya, K. Kamalanand, V. Rajinikanth, R.S. Nayak, and S. Kadry, "Deep Neural Network Assisted Diagnosis Of Time-Frequency Transformed Electromyograms,” Multimedia Tools and Applications, vol.79, no.15, pp.1105111067, 2020. DOI: https://doi.org/10.1007/s11042-018-6561-9

[5] L.A.Lund, Z. Omar, I. Khan, S. Kadry, S. Rho, I.A., Mari, and K.S.Nisar, "Effect Of Viscous Dissipation in Heat Transfer of MHD Flow of Micropolar Fluid Partial Slip Conditions: Dual Solutions and Stability Analysis,” Energies, vol.12, no.24, pp.4617, 2019. DOI: https://doi.org/10.3390/en12244617

[6] S. Cotsakis, S. Kadry, G. Kolionis, and A. Tsokaros, "Asymptotic Vacua With Higher Derivatives," Physics Letters B, vol.755, pp.387-392, 2016. DOI: https://doi.org/10.1016/j.physletb.2016.02.036

[7] K. Smaili, T. Kadri, and S. Kadry, "A Modified-Form Expressions for the Hypoexponential Distribution," British Journal of Mathematics \& Computer Science, vol.4, no.3, pp.322-332, 2014 . DOI: https://doi.org/10.9734/BJMCS/2014/6317

[8] A. Abou Jaoude, E.T. Khaled, S. Kadry, and H. Noura, "Prognostic Model for Buried Tubes," Journal of Mathematics and Statistics, vol.6, no.2, pp.116-124, 2010. DOI: https://doi.org/10.3844/jmssp.2010.116.124

[9] V. Rajinikanth, S.C. Satapathy, N. Dey, and R. Vijayarajan,.., "DWT-PCA Image Fusion Technique to Improve Segmentation Accuracy in Brain Tumor Analysis," Lecture Notes in Electrical Engineering," vol.471, pp.453-462, 2018. DOI: https://doi.org/10.1007/978-981-10-7329-8_46.

[10] V. Rajinikanth, N.S.M. Raja, and S.C. Satapathy, "Robust Color Image Multi-Thresholding Using Between-Class Variance and Cuckoo Search Algorithm," Advances in Intelligent Systems and Computing, vol.433, pp. 379-386, 2016. DOI: https://doi.org/10.1007/978-81-322-2755-7_40

[11] S.L. Fernandes, V. Rajinikanth, and S. Kadry, "A Hybrid Framework to Evaluate Breast Abnormality Using Infrared Thermal Images," IEEE Consumer Electronics Magazine," vol.8, no.5, pp.31-36, 2019. https://doi.org/10.1109/MCE.2019.2923926

[12] S.L.Fernandes, U.J.Tanik, V. Rajinikanth, and K.A. Karthik, "A Reliable Framework for Accurate Brain Image Examination and Treatment Planning Based on Early Diagnosis Support For Clinicians," Neural Computing and Applications, vol.32, no.20, pp.15897-15908, 2020. DOI: https://doi.org/10.1007/s00521-019-04369-5

[13] S.C. Satapathy and V.Rajinikanth, "Jaya Algorithm Guided Procedure to Segment Tumor From Brain MRI," Journal of Optimization, vol. 2018, ID 3738049, 2018. DOI: https://doi.org/10.1155/2018/3738049

[14] T.V. Shree, K. Revanth, N.S.M. Raja, and V. Rajinikanth, "A Hybrid Image Processing Approach to Examine Abnormality in Retinal Optic Disc," Procedia Computer Science, vol.125, pp.157-164, 2018. DOI: https://doi.org/10.1016/j.procs.2017.12.022

[15] V. Rajinikanth, N.S.M. Raja, and N. Dey, A Beginner's Guide to Multilevel Image Thresholding, $1^{\text {st }}$ Edition, CRC Press, 2020. DOI: https://doi.org/10.1201/9781003049449-1 
[16] S. Kadry, G. Alferov, G. Ivanov, A. Sharlay "Almost Periodic Solutions of First-Order Ordinary Differential Equations," Mathematics, vol.6, no.9, pp.171, 2018. DOI: https://doi.org/10.3390/math6090171

[17] N.S.M. Raja, K.S. Manic, and V. Rajinikanth, "Firefly Algorithm with Various Randomization Parameters: An Analysis," Lecture Notes in Computer Science, vol.8297, pp.110-121, 2013. DOI: https://doi.org/10.1007/978-3319-03753-0_11

[18] V. Rajinikanth, K. Kamalanand,C. Emmanuel, and B. Thayumanavan, Biomedical Signal and Image Examination with Entropy-Based Techniques, $1^{\text {st }}$ edition, CRC Press, 2020. DOI: https://doi.org/10.1201/9780367477240-1

[19] A. Ahilan, G. Manogaran,C. Raja, S.Kadry, S.N.Kumar, C.A. Kumar,T. Jarin, S. Krishnamoorthy, P.M. Kumar, G.C., Babu, and N.S. Murugan, "Segmentation by Fractional Order Darwinian Particle Swarm Optimization Based Multilevel Thresholding and Improved Lossless Prediction Based Compression Algorithm for Medical Images," IEEE Access, vol.7, pp.89570-89580, 2019. DOI: https://doi.org/10.1109/ACCESS.2019.2891632

[20] S. Agrawal, R. Panda, S. Bhuyan, and B.K. Panigrahi, "Tsallis Entropy Based Optimal Multilevel Thresholding Using Cuckoo Search Algorithm," Swarm and Evolutionary Computation, vol.11, pp.16-30., 2013. DOI: https://doi.org/10.1016/j.swevo.2013.02.001

[21] Y. Zhang, and L. Wu, "Optimal Multi-Level Thresholding Based on Maximum Tsallis Entropy Via an Artificial Bee Colony Approach," Entropy, vol.13, no.4, pp.841-859, 2011. DOI: https://doi.org/10.3390/e13040841

[22] Y. Wang, G. Zhang, and X. Zhang, "Multilevel Image Thresholding Using Tsallis Entropy and Cooperative Pigeoninspired Optimization Bionic Algorithm," Journal of Bionic Engineering, vol.16, no.5, pp.954-964, 2019. DOI: https://doi.org/10.1007/s42235-019-0109-1

[23] K.S. Manic, R.K. Priya, and V. Rajinikanth, "Image multithresholding based on Kapur/Tsallis entropy and firefly algorithm. Indian Journal of Science and Technology,” vol. 9, no.12, pp.89949, 2016. DOI: https://doi.org/10.17485/ijst/2016/v9i12/89949

[24] H.S. Naji Alwerfali, M.A. Al-qaness, M. Abd Elaziz, M., A.A. Ewees,D. Oliva, and S. Lu, "Multi-Level Image Thresholding Based on Modified Spherical Search Optimizer and Fuzzy Entropy,” Entropy, vol.22, no.3, pp.328, 2020. DOI: https://doi.org/10.3390/e22030328

[25] A.K.M. Khairuzzaman and S. Chaudhury, "Moth-Flame Optimization Algorithm Based Multilevel Thresholding for Image Segmentation,” International Journal of Applied Metaheuristic Computing (IJAMC), vol.8, no.4, pp.58-83, 2017. DOI: https://doi.org/10.4018/IJAMC.2017100104

[26] M. Abd El Aziz, A. A. Ewees, and A.E. Hassanien, "Whale Optimization Algorithm and Moth-Flame Optimization for Multilevel Thresholding Image Segmentation,” Expert Systems with Applications, vol.83, pp.242-256, 2017. DOI: https://doi.org/10.1016/j.eswa.2017.04.023

[27] H. Jia, J. Ma, and W. Song, "Multilevel Thresholding Segmentation for Color Image Using Modified Moth-Flame Optimization,” IEEE Access, vol.7, pp.44097-44134, 2019. DOI: https://doi.org/10.1109/ACCESS.2019.2908718

[28] A.E. Hassanien, T. Gaber, U. Mokhtar, and H. Hefny, "An Improved Moth Flame Optimization Algorithm Based on Rough Sets for Tomato Diseases Detection," Computers and Electronics in Agriculture, vol.136, pp.86-96, 2017. DOI: https://doi.org/10.1016/j.compag.2017.02.026

[29] C. Tsallis, "Possible Generalization of Boltzmann-Gibbs Statistics," Journal of statistical physics, vol.52, no.1-2, pp.479-487, 1988. DOI: https://doi.org/10.1007/BF01016429

[30] V. Rajinikanth and M.S. Couceiro, "Optimal Multilevel Image Threshold Selection Using A Novel Objective Function," In Information Systems Design and Intelligent Applications, pp. 177-186, 2015. DOI: https://doi.org/10.1007/978-81-322-2247-7_19

[31] S. Mirjalili, "Moth-Flame Optimization Algorithm: A Novel Nature-Inspired Heuristic Paradigm,” Knowledge-based systems, vol.89, pp.228-249, 2015. DOI: https://doi.org/10.1016/j.knosys.2015.07.006

[32] V. Rajinikanth, N. Dey, E. Kavallieratou, and H. Lin, "Firefly Algorithm-Based Kapur's Thresholding and Hough Transform to Extract Leukocyte Section from Hematological Images," In Applications of Firefly Algorithm and its Variants, pp. 221-235, Springer, Singapore., 2020. DOI: https://doi.org/10.1007/978-981-15-0306-1_10 